\title{
Quantifying crack tip displacement fields with DIC
}

\author{
J.R. Yates, M. Zanganeh, Y.H. Tai * \\ Dept. of Mechanical Engineering, University of Sheffield, Mappin Street, S1 3JD, UK
}

\section{A R T I C L E I N F O}

\section{Article history:}

Received 18 December 2009

Received in revised form 12 March 2010

Accepted 16 March 2010

Available online 27 March 2010

\section{Keywords:}

Crack tip displacement fields

Digital image correlation

K

T-stress

CTOA

\begin{abstract}
A B S T R A C T
Crack paths under both fatigue and fracture conditions are governed by the crack tip displacement field and the material deformation characteristics, including those influenced by metallurgical anisotropy. Experimental techniques such as thermoelasticity and photoelasticity have been successfully used to characterise the elastic stress fields around cracks but they do not take into account either plasticity or anisotropy. Considerable work has been carried out to characterise crack tip stress fields from displacement measurements. The current method of choice for obtaining displacement field data is digital image correlation (DIC) which has undergone significant advances in the recent years. The ease of use and capabilities of the technique for full field displacements has led to improved methods for characterising crack tip displacement fields based on data obtained from DIC. This paper gives an overview of some of the applications of DIC for crack tip characterisation such as $K, T$-stress and crack tip opening angle (CTOA) measurements as well as data obtained from 3D measurements of a propagating crack.
\end{abstract}

(c) 2010 Elsevier Ltd. All rights reserved.

\section{Introduction}

The path that a crack follows, whether it is under cyclic or monotonic loading, is strongly influenced by the elastic and plastic displacement field at the crack tip. These displacements are governed by a combination of material deformation behaviour, the mechanics of the loading and the geometry of the structure [1].

For many years, our investigations into crack paths, has been based on characterising the crack path displacement field through the elastic component. Photoelastic stress analysis, thermoelaticity, caustics, Moire interferometry and many other have been used to successfully characterise the elastic stress fields around cracks but these techniques are not able to take into consideration effects of plasticity or anisotropy [2]. This has led to considerable work on developing techniques for characterising crack tip stress fields from displacement field measurements.

The current method of choice for characterising displacement fields is digital image correlation (DIC) which is a relatively straight forward and cost effective technique. With the recent availability of high quality, high resolution digital cameras as well as the development of image correlation algorithms, it has enabled the possibility for full field elastic-plastic displacement field to be measured directly. This has opened up the opportunity of exploring the influence of plastic deformations around the crack tip on the growing crack.

In this paper, an overview of some the possible applications of DIC for crack tip characterisation will be presented. This will include $K$ and $T$-stress [3,4] characterisation for fatigue cracks in double cantilever beam (DCB) specimens, crack closure effects on measured $K$ using CT specimens, CTOA characterisation from displacement field data as well as 3D full field displacement data for tearing cracks in an anisotropic material.

\footnotetext{
* Corresponding author. Tel.: +44 1142227892; fax: +44 1142227890 .

E-mail address: y.tai@sheffield.ac.uk (Y.H. Tai).
} 


\section{Basic principles of digital image correlation}

A digital image is essentially a two-dimensional array of intensity values which can be discretized into small subsets. Image correlation works by matching small square subsets of an undeformed image to locations in the image of the surface after deformation as illustrated in Fig. 1 by means of a series of mathematical mapping and cross correlation functions. For this technique to work well a grey scale random pattern is needed on the surface of the specimen. To recognize this pattern mathematically, the intensity of each pixel in the reference and deformed images can be traced and the displacement vector can be determined. However, it would be extremely difficult to distinguish every single pixel on the image and therefore at least $3 \times 3$ pixels are needed for one recognizable feature. The ideal subset size should contain at least three clear features but it is often a compromise between resolution and accuracy. As a general rule, larger subset sizes will increase the accuracy whereas a smaller subset will increase the resolution but realistically the size of a subset is determined by the quality of the image and speckle pattern.

A variety of methods can be used to produce a random pattern on the surface. Sometimes the natural pattern of the material is enough to produce a suitable pattern. Glass or emery paper can be used to scratch the surface of the specimen to generate a random pattern [5]. More conventionally, the random pattern is produced by spray painting the surface or using dry toner with an adhesive medium. These techniques normally suffice for macro digital image correlation applications but for micro scale applications more care is needed when producing the random pattern due to the speckle size and often requires the use of an airbrush or lithography. Fig. 2 illustrates the typical set-up of a 2D DIC. A 3D system will have two cameras at different angles to obtain a 3D perspective of the specimen surface.

\subsection{Displacement mapping}

Assume that a point $P$ in the reference image with an $x$ - and $y$-coordinate system is mapped into point $P^{*}$ in deformed image with an $x^{*}$ and $y^{*}$ coordinate system (Fig. 3 ). The mapping can be performed as,

$$
\begin{aligned}
& x^{*}=x+u(x, y) \\
& y^{*}=y+v(x, y)
\end{aligned}
$$

To find the displacement fields, the vertical, $v$, and horizontal, $u$, displacements can be approximated using the Taylor series around a point $P\left(x_{0}, y_{0}\right)$ as,

$$
\begin{aligned}
& x^{*}=x_{0}+u_{0}+\frac{\partial u}{\partial x} \Delta x+\frac{\partial u}{\partial y} \Delta y+\frac{1}{2} \frac{\partial^{2} u}{\partial x^{2}} \Delta x^{2}+\frac{1}{2} \frac{\partial^{2} u}{\partial y^{2}} \Delta y^{2}+\frac{\partial^{2} u}{\partial x \partial y} \Delta x \Delta y \\
& y^{*}=y_{0}+v_{0}+\frac{\partial v}{\partial x} \Delta x+\frac{\partial v}{\partial y} \Delta y+\frac{1}{2} \frac{\partial^{2} v}{\partial x^{2}} \Delta x^{2}+\frac{1}{2} \frac{\partial^{2} v}{\partial y^{2}} \Delta y^{2}+\frac{\partial^{2} v}{\partial x \partial y} \Delta x \Delta y
\end{aligned}
$$

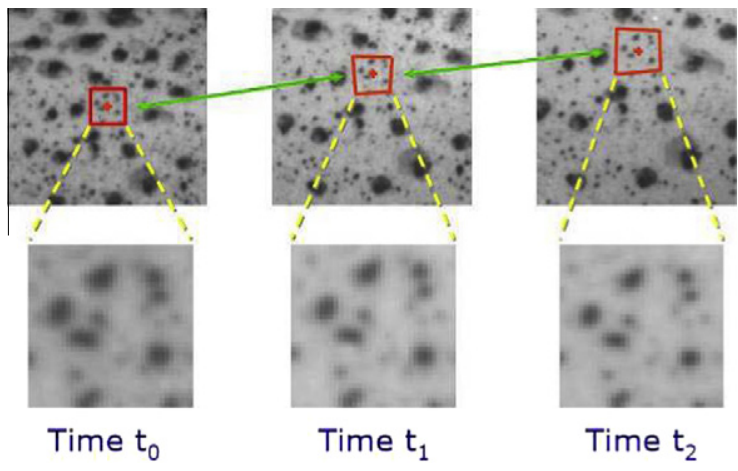

Fig. 1. Illustration of subset matching and deformation tracking used for DIC (Image courtesy of Limess GmbH).

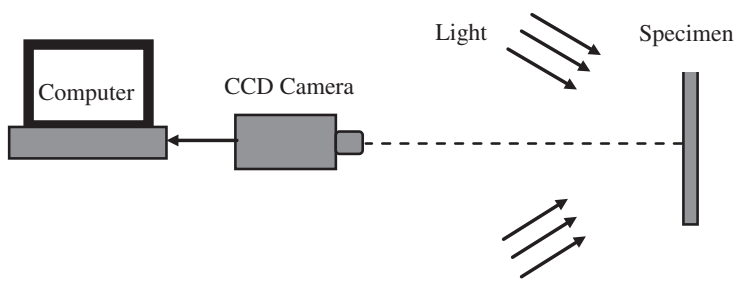

Fig. 2. Schematic diagram of typical 2D DIC equipment. 


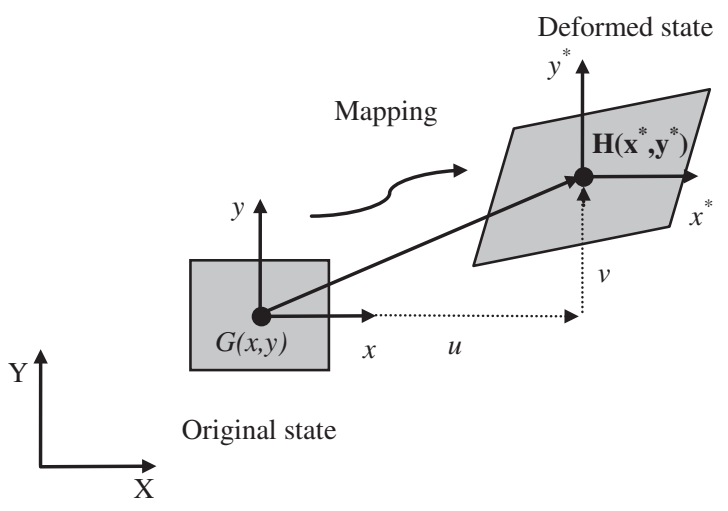

Fig. 3. Concept of digital image correlation.

in which $\Delta x=x-x_{0}$ and $\Delta y=y-y_{0}$.

Depending on the use of a first or second order approximation, 6 or 12 unknowns are available and can be found by correlation between the two images. This can be done by minimizing the so called correlation factor, $C$, defined as,

$$
C=\frac{\sum_{s}\left[G(x, y)-H\left(x^{*}, y^{*}\right)\right]^{2}}{\sum_{s} G^{2}(x, y)}
$$

where $G$ and $H$ are the grey scale light intensities corresponding to all the points in the subset, $S$. Since the intensity of light obtained from a digital image is a digital quantity, it needs to be smoothed first and then be used in the cross correlation algorithm. This can be done using B-Splines, Bi-cubic splines or any other interpolation technique. As an alternative, a Fast Fourier Transform (FFT) cross correlation can be used to compare the subsets.

\section{Applications}

\subsection{Evaluation of stress intensity factor and T-stress}

There are two different approaches to tackle any elasticity problem using experimental data. The first approach is to guess a general form of analytical function and fit this to the experimental data and then determine the displacement field and stress field. Muskhelishvili's [6] approach belongs to this group in which two complex analytical functions need to be used.

The second approach is to guess an analytical stress function, it can be complex or not, satisfying the boundary conditions and determining the displacement and stress field analytically. These analytical fields can be fitted to the experimental data and the required parameters, for example, $T$-stress and stress intensity factor, can be determined. One such analytical solution is Williams' asymptotic formulation [7]. In this work Williams' approach is used to extract the $T$-stress and $K_{\mathrm{I}}, K_{\mathrm{II}}$ from experimental displacement data obtained from DIC measurements.

\subsubsection{Methodology (Williams' approach)}

The displacement field ahead of a crack can be expressed as an infinite series [8,9]. In a plane mixed mode I and II condition this displacement field is expressed as

$$
\text { Mode I }\left\{\begin{array}{l}
u_{\mathrm{I}}=\sum_{n=1}^{\infty} \frac{r^{\frac{n}{2}}}{2 \mu} a_{n}\left\{\left[\kappa+\frac{n}{2}+(-1)^{n}\right] \cos \frac{n \theta}{2}-\frac{n}{2} \cos \frac{(n-4) \theta}{2}\right\} \\
v_{\mathrm{I}}=\sum_{n=1}^{\infty} \frac{r^{2}}{2 \mu} a_{n}\left\{\left[\kappa-\frac{n}{2}-(-1)^{n}\right] \sin \frac{n \theta}{2}+\frac{n}{2} \sin \frac{(n-4) \theta}{2}\right\}
\end{array}\right.
$$

and,

$$
\text { Mode II }\left\{\begin{array}{l}
u_{\mathrm{II}}=-\sum_{n=1}^{\infty} \frac{r^{2}}{2 \mu} b_{n}\left\{\left[\kappa+\frac{n}{2}-(-1)^{n}\right] \sin \frac{n \theta}{2}-\frac{n}{2} \sin \frac{(n-4) \theta}{2}\right\} \\
v_{\mathrm{II}}=\sum_{n=1}^{\infty} \frac{r^{\frac{n}{2}}}{2 \mu} b_{n}\left\{\left[\kappa-\frac{n}{2}+(-1)^{n}\right] \cos \frac{n \theta}{2}+\frac{n}{2} \cos \frac{(n-4) \theta}{2}\right\}
\end{array}\right.
$$

where $u$ and $v$ are horizontal ( $x$-direction) and vertical ( $y$-direction) displacements in mode I and II. $\mu$ is the shear modulus and $\kappa=(3-v) /(1+v)$ for plane stress and $\kappa=3-4 v$ for plane strain conditions, where $v$ is the Poisson's ratio. $a$ and $b$ are constants and $r$ and $\theta$ are radial and phase distance from crack tip. 
Mixed mode displacement fields ( $u$ and $v$ ) which are obtained from DIC, can be derived by superimposing the mode I and II displacement fields. By defining $f_{n, m}(r, \theta), g_{n, m}(r, \theta), h_{n, m}(r, \theta)$ and $l_{n, m}(r, \theta)$ as follows (Eq. (6)), the displacement field (Eqs. (4) and (5)) can be written in a matrix form (Eq. (7))

$$
\begin{gathered}
f_{n, m}=\frac{r_{m}^{\frac{n}{2}}}{2 \mu}\left\{\left[\kappa+\frac{n}{2}+(-1)^{n}\right] \cos \frac{n \theta_{m}}{2}-\frac{n}{2} \cos \frac{(n-4) \theta_{m}}{2}\right\} \\
g_{n, m}=\frac{-r_{m}^{\frac{n}{2}}}{2 \mu}\left\{\left[\kappa+\frac{n}{2}-(-1)^{n}\right] \sin \frac{n \theta_{m}}{2}-\frac{n}{2} \sin \frac{(n-4) \theta_{m}}{2}\right\} \\
h_{n, m}=\frac{r_{m}^{\frac{n}{2}}}{2 \mu}\left\{\left[\kappa-\frac{n}{2}-(-1)^{n}\right] \sin \frac{n \theta_{m}}{2}+\frac{n}{2} \sin \frac{(n-4) \theta_{m}}{2}\right\} \\
l_{n, m}=\frac{r_{m}^{\frac{n}{2}}}{2 \mu}\left\{\left[\kappa-\frac{n}{2}+(-1)^{n}\right] \cos \frac{n \theta_{m}}{2}+\frac{n}{2} \cos \frac{(n-4) \theta_{m}}{2}\right\} \\
\left\{\begin{array}{c}
u_{1} \\
\vdots \\
u_{m} \\
v_{1} \\
\vdots \\
v_{m}
\end{array}\right\}=\left[\begin{array}{cc}
f_{1,1} \cdots f_{n, 1} & g_{1,1} \cdots g_{n, 1} \\
\vdots & \vdots \\
f_{1, m} \cdots f_{n, m} & g_{1, m} \cdots g_{n, m} \\
h_{1,1} \cdots h_{n, 1} & l_{1,1} \cdots l_{n, 1} \\
\vdots & \vdots \\
h_{1, m} \cdots h_{n, m} & l_{1, m} \cdots l_{n, m}
\end{array}\right]\left\{\begin{array}{c}
a_{1} \\
\vdots \\
a_{n} \\
b_{1} \\
\vdots \\
b_{n}
\end{array}\right\}
\end{gathered}
$$

where $m$ is the data point index.

By expanding Eqs. (4) and (5), omitting the terms of order $r^{3 / 2}$ and above and comparing with the more common notation, using stress intensity factors and $T$-stress as shown in the following equation,

$$
\begin{aligned}
& u=\frac{K_{\mathrm{I}}}{2 \mu} \sqrt{\frac{r}{2 \pi}} \cos \frac{\theta}{2}\left(\kappa-1+2 \sin ^{2} \frac{\theta}{2}\right)+\frac{K_{\mathrm{II}}}{2 \mu} \sqrt{\frac{r}{2 \pi}} \sin \frac{\theta}{2}\left(\kappa+1+2 \cos ^{2} \frac{\theta}{2}\right)+\frac{T}{8 \mu} r(\kappa+1) \cos \theta \\
& v=\frac{K_{\mathrm{I}}}{2 \mu} \sqrt{\frac{r}{2 \pi}} \sin \frac{\theta}{2}\left(\kappa+1-2 \cos ^{2} \frac{\theta}{2}\right)-\frac{K_{\mathrm{II}}}{2 \mu} \sqrt{\frac{r}{2 \pi}} \cos \frac{\theta}{2}\left(\kappa-1-2 \cos ^{2} \frac{\theta}{2}\right)+\frac{T}{8 \mu} r(\kappa-3) \sin \theta
\end{aligned}
$$

it can be shown that

$$
K_{\mathrm{I}}=a_{1} \sqrt{2 \pi}, \quad K_{\mathrm{II}}=-b_{1} \sqrt{2 \pi}, \quad T=4 a_{2}
$$

in which $K_{\mathrm{I}}$ is the mode I stress intensity factor, $K_{\mathrm{II}}$ is mode II stress intensity factor and $T$ is the $T$-stress.

Looking at Eq. (7) it is evident that no rigid body motion term has been considered in that equation. This can be included by adding constant terms in Eq. (7) to compensate for the rigid body translations and another term to compensate for the rigid body rotation as follows:

$$
\left\{\begin{array}{c}
u_{1} \\
\vdots \\
u_{m} \\
v_{1} \\
\vdots \\
v_{m}
\end{array}\right\}=\left[\begin{array}{ccccc}
1 & f_{1,1} \cdots f_{n, 1} & 0 & g_{1,1} \cdots g_{n, 1} & -r_{1} \sin \theta_{1} \\
\vdots & \vdots & \vdots & \vdots & \vdots \\
1 & f_{1 m} \cdots f_{n, m} & 0 & g_{1, m} \cdots g_{n, m} & -r_{m} \sin \theta_{m} \\
0 & h_{1,1} \cdots h_{n, 1} & 1 & l_{1,1} \cdots l_{n, 1} & r_{1} \sin \theta_{1} \\
\vdots & \vdots & \vdots & \vdots & \vdots \\
0 & h_{1, m} \cdots h_{n, m} & 1 & l_{1, m} \cdots l_{n, m} & r_{m} \cos \theta_{m}
\end{array}\right]\left\{\begin{array}{c}
a_{0} \\
a_{1} \\
\vdots \\
a_{n} \\
b_{0} \\
b_{1} \\
\vdots \\
b_{n} \\
R
\end{array}\right\}
$$

where $a_{0}$ and $b_{0}$ are used to compensate for the rigid body motion and $R$ compensates for the rigid body rotation.

It should be noted that in solution of Eq. (10), for each value on $n, a_{0}$ to $a_{n}$ are determined and the stress intensity factors and the $T$-stress are calculated using Eq. (9). It is evident that for $n=1$ only stress intensity factors are determinable. For $n=2$ both intensity factors and the $T$-stress can be determined. However, the displacement reconstructed based on these calculated SIFs and the $T$-stress (for $n=2$ ) is not necessarily the best fit to the experimental displacement fields. Therefore, it is required to increase the number of terms $(n)$ and recalculate the SIFs and the $T$-stress for each value of $n$. There is no definite answer to the required number of terms to obtain the best fit for the experimental data for all the applications. Hence, the number of terms should be increased until no considerable changes in determined parameters or a given correlation function between experimental fields and predicted fields are observed. 


\subsubsection{Experimental results}

Experiments were undertaken on fatigue cracks emanating from notches to collect crack tip field displacement data. Pure mode I loading condition was created ahead of a $4 \mathrm{~mm}$ notch in a $5 \mathrm{~mm}$ thick DCB specimen with the dimensions shown in Fig. 4.

The specimens were machined from a plate of $7010 \mathrm{~T} 7651$ aluminium alloy and spark eroded to introduce the notch into the specimen. The required speckles for DIC were produced on the surface of specimen using a fine spray of matt black paint. A $100 \mathrm{kN}$ MAND hydraulic test machine was used to load the specimen in tension. A 14 bit, $1600 \times 1200 \mathrm{CCD}$ camera and a Nikon lens with a resolution of 18.75 microns per pixel was used to record the images. DaVis software [10] was employed to correlate the images. Fig. 5 shows a typical correlated displacement field obtained around a crack. The experiments were continued by growing a fatigue crack from the notch tip, using a $0.5 \mathrm{kN}$ to $2 \mathrm{kN}$ load range at a loading frequency of $15 \mathrm{~Hz}$. The crack growth was paused when the fatigue crack length was approximately $1 \mathrm{~mm}, 4 \mathrm{~mm}, 8 \mathrm{~mm}$ and $15 \mathrm{~mm}$. For each increment of crack growth load ranges of $0.5-1.5 \mathrm{kN}$ and $0.5-3 \mathrm{kN}$ were applied, images were recorded at each load and processed to determine the stress intensity factors and $T$-stress. It should be noted that the size plastic zone corresponding to the highest load range is in order of $30 \mu \mathrm{m}$ which is very small in comparison with the measurements made.

The displacement field obtained from DIC was imported to the DICITAC software [11] to determine the stress intensity factors and the T-stress using Eqs. (5) and (6) under plane stress conditions. In the solution process of Eq. (9), the number of terms was increased until the stress intensity factors and the T-stress converged as typically shown in Fig. 6 for the $8 \mathrm{~mm}$ fatigue crack example. These figures also show the quality of the fitted data to the experimental displacement fields. The fitted data shown in Fig. $6 a$ and $\mathrm{b}$ are based on the recreation of the displacement fields using the coefficients from 15 terms of Williams' solution.

In numerical simulation of the problem, an elastic model as shown in Fig. 7 was solved using ABAQUS [12]. Quarter point singular elements used to model the elastic singularity ahead of the crack tip and the stress intensity factors were determined using the $J$-integral method. The $T$-stress was also determined using an interaction integral technique. More details on extraction of mixed mode SIFs and T-stress using $J$-integral method and interaction integral technique can be found in ABAQUS reference manual [12].

As it can be observed from Figs. 8 and 9, although the values found for the mode II stress intensity factors using the 3 term solution are slightly better than the others, using any of the solutions yields practically zero values for mode II stress intensity factors in both the $0.5-1.5 \mathrm{kN}$ and $0.5-3 \mathrm{kN}$ load ranges.

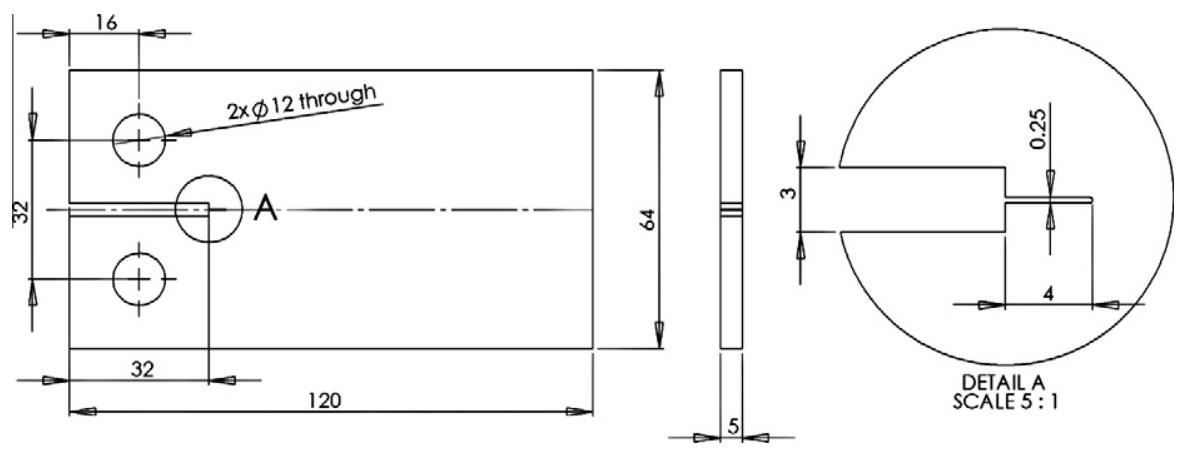

Fig. 4. DCB specimen dimensions in $\mathrm{mm}$.

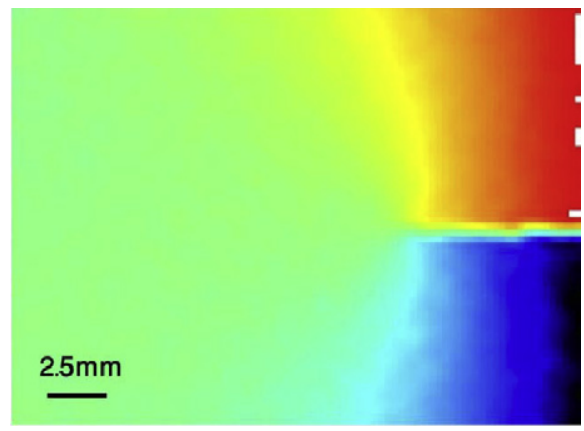

Vertical displacement

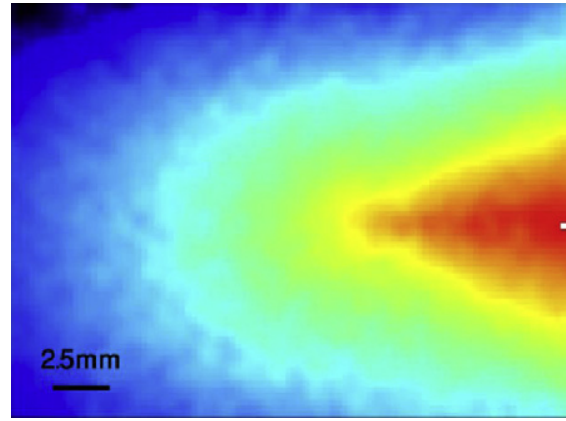

Horizontal displacement

Fig. 5. Typical correlated displacement fields obtained around a crack using DIC. 

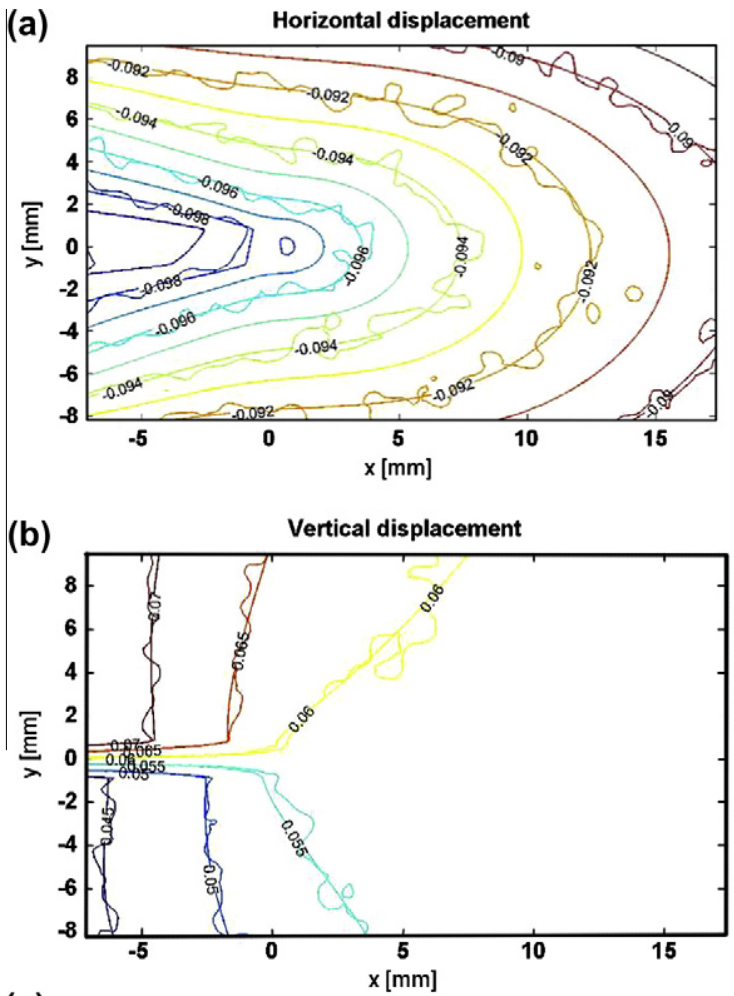

(c)

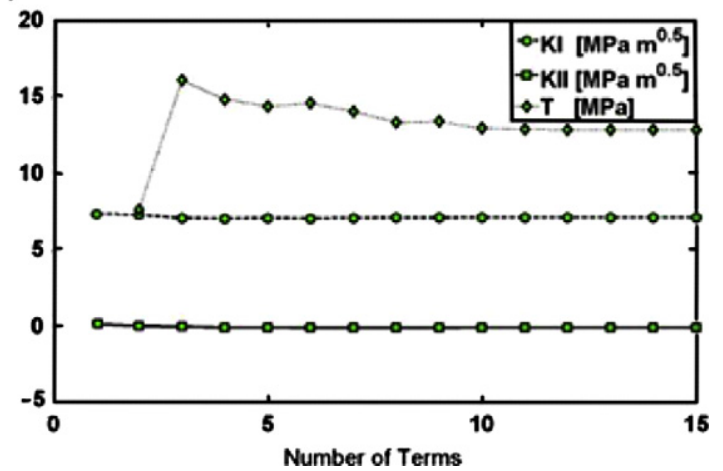

Fig. 6. Quality of fitted displacement field: (a) horizontal and (b) vertical and (c) variation of T-stress and SIF versus number of Williams' solution terms for the $8 \mathrm{~mm}$ fatigue crack under $0.5-1.5 \mathrm{kN}$ loading condition.

Experimentally determined mode I stress intensity factors are in good agreement with the numerically simulated cases, see Fig. 8a. The average differences were 12.6\% (with standard deviation of 8.8\%) using the 3 term solution and $12.9 \%$ (with standard deviation of 11.5\%) using the converged 15 term solution. Part of the difference in the experimental results compared to FE results is due to the errors introduced into the correlated displacement field because of a low signal to noise ratio. Looking at Fig. 9a in which the load range has increased from 0.5 to $3 \mathrm{kN}$ the average difference decreased to $12.1 \%$ (with standard deviation of $6.2 \%$ ) using the 3 term solution, $12.9 \%$ (with standard deviation of $6.9 \%$ ) using the converged 15 term solution.

Almost the same trend as the mode I stress intensity factors is observed (Fig. 8b) for the experimentally determined $T$ stress when compared to the FE results. The average differences of $24.9 \%$ (with standard deviation of $2.2 \%$ ) and $35.4 \%$ (with standard deviation of $14.9 \%$ ) were found using 3 term and converged 15 term solutions, respectively for $0.5-1.5 \mathrm{kN}$ load range. In $0.5-3 \mathrm{kN}$ load range case (Fig. 9b), the average differences were found as to be $14.4 \%$ (with standard deviation of $8.9 \%$ ), $36.0 \%$ (with standard deviation of $27.4 \%$ ) and $24.9 \%$ (with standard deviation of $23.2 \%$ ) using the 3 term, converged solutions, respectively.

The agreement between the numerical and experimental results obtained for the $T$-stress is not as good as for the stress intensity factor results. Compared to the stress intensity factor, the $T$-stress is the coefficient of higher order terms of the 


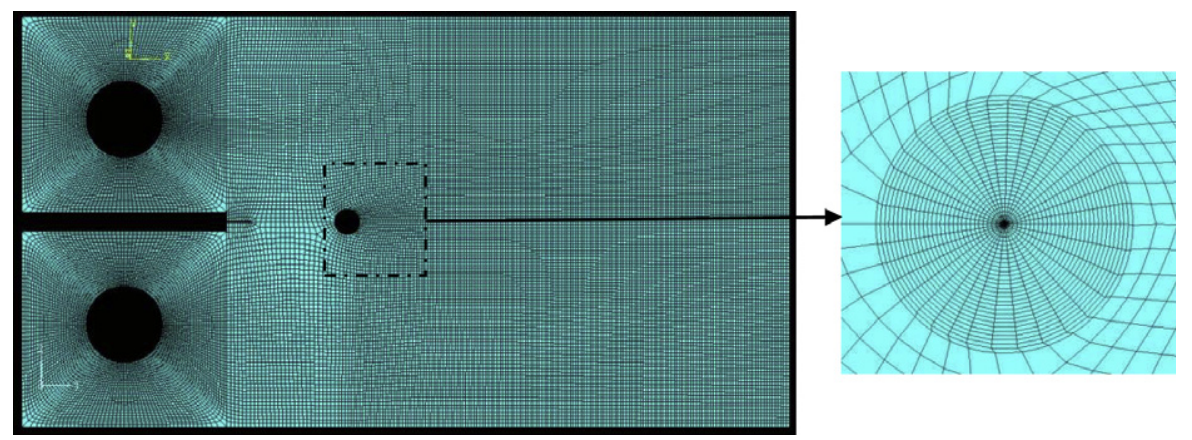

Fig. 7. FE model created in ABAQUS.

displacement field expansions, this makes the $T$-stress relatively a more difficult parameter to measure experimentally. Nevertheless, some of the discrepancy is likely to be due to the limitations of the finite element model. The realities of a nonplanar crack, crack front curvature, surface residual stresses from machining, material anisotropy and crack closure will all compromise the modelling. The effect of latter one, crack closure, will be investigated in the next section. Given the consistency and smooth trend in the experimental $T$-stress values, the proposed methodology provides a reliable and robust technique for determining elastic constraint in fatigue and fracture testing.

\subsection{Effects of crack closure}

The effects of crack closure are well known and has been characterised using Mushkhelishvili's approach and DIC data before by other researchers $[5,13]$. This section demonstrates the capability of the Williams' approach as shown in the previous section and 3D DIC for characterising the effects of crack closure on the stress fields using an aluminium lithium compact tension specimen undergoing fatigue loading.

\subsubsection{Methodology}

A compact tension specimen made from a 2195-T 8 aluminium lithium alloy in the LT direction was used. Specimen dimensions were in accordance to the guidelines found in the ASTM-E2472 standard [14] and are illustrated in Fig. 10. The notch was machined by electrode discharge machining (EDM) and the resultant notch length gave a crack length ratio
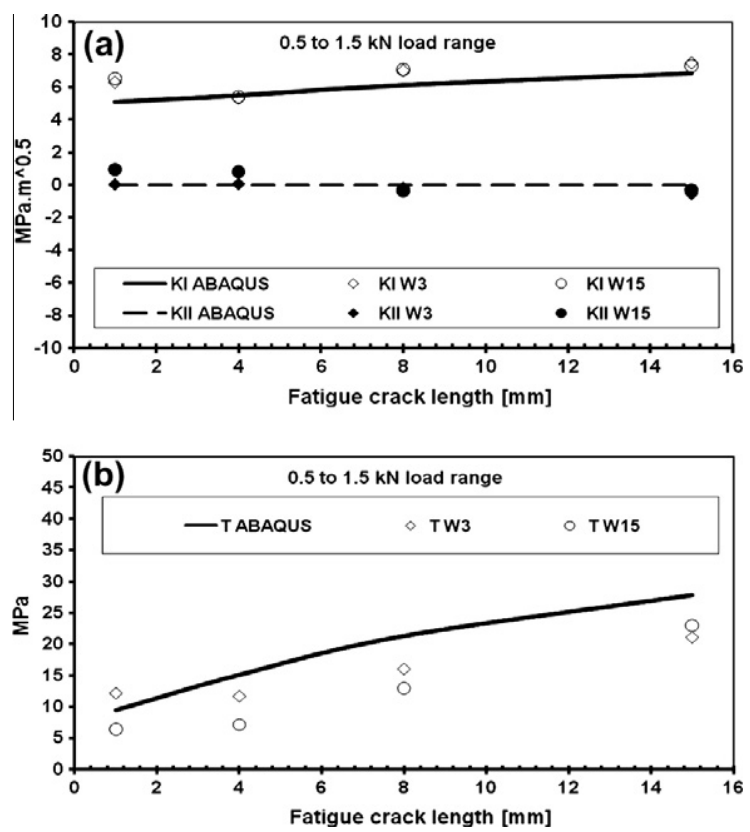

Fig. 8. Comparison of (a) stress intensity factors and (b) T-stresses determined using ABAQUS, 3 term Williams (W3) and 15 term Williams (W15) for 0.5$1.5 \mathrm{kN}$ load range. 

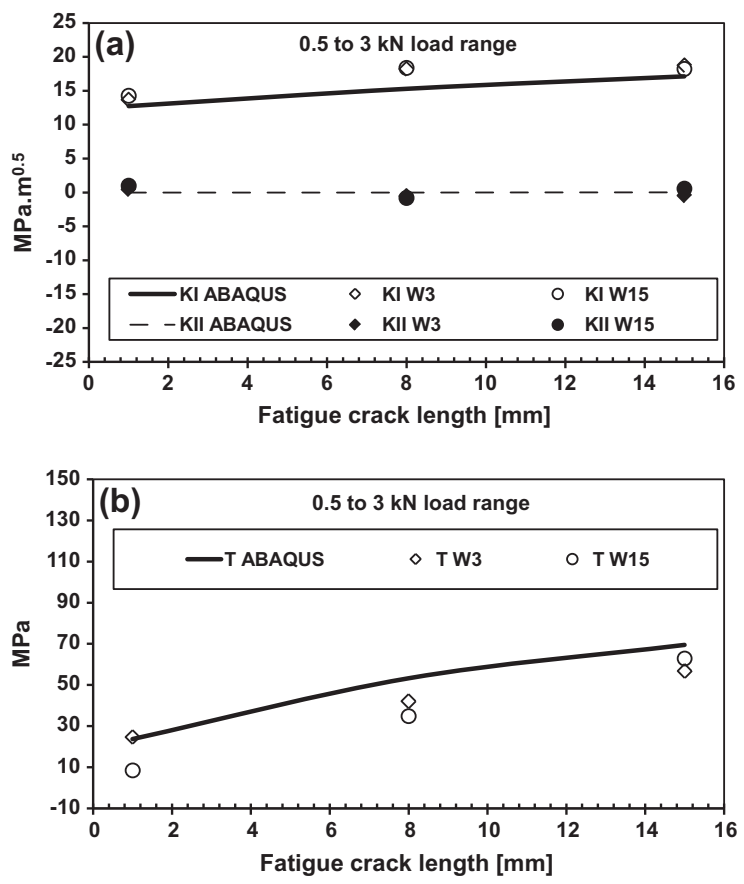

Fig. 9. Comparison of (a) stress intensity factors and (b) T-stresses determined using ABAQUS, 3 term Williams (W3) and 15 term Williams (W15) for 0.5$3 \mathrm{kN}$ load range.

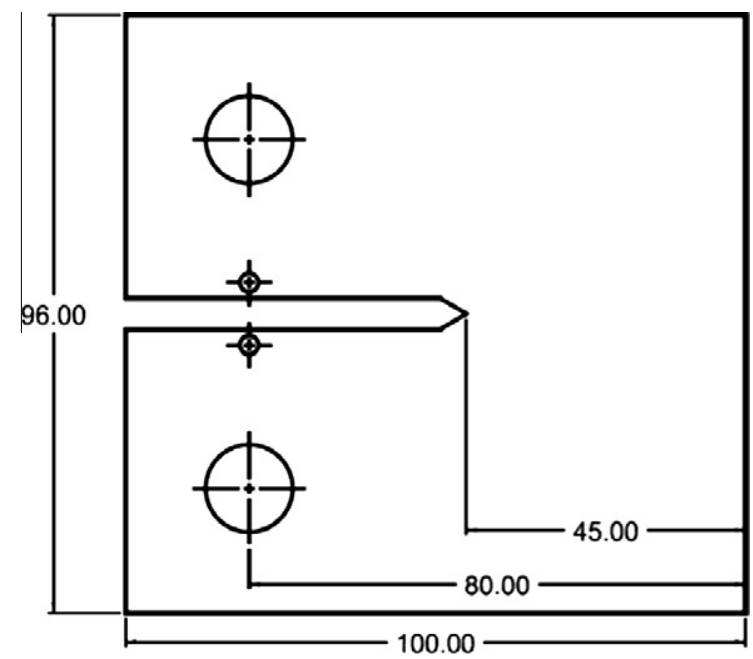

Fig. 10. Schematic diagram of $\mathrm{CT}$ specimen, dimensions in $\mathrm{mm}$.

to specimen width of 0.44 . This allowed for approximately $20 \mathrm{~mm}$ of geometry independent crack extension. A 3D DIC system with a pair of 5MP monochrome cameras was used to for measuring the displacement field of the specimen surface which was prepared with a speckle pattern. Fatigue cracking was carried out in a servo hydraulic test machine under mode I conditions at a $R$ ratio $=0.1$ and a $\Delta K=15 \mathrm{MPa} \sqrt{ } \mathrm{m}$ at the beginning of the test. Loading frequency was at $10 \mathrm{~Hz}$ during the crack growth stages but the process was paused at intervals to allow for measurements to be taken at the various stages of crack growth.

\subsubsection{Experimental results}

The results from $e_{y y}$ logarithmic strains calculated using the DIC for a $10 \mathrm{~mm}$ crack when no loading was applied is shown in Fig. 11. The line originating from the machined notch illustrates the approximate location of the crack and crack length. 


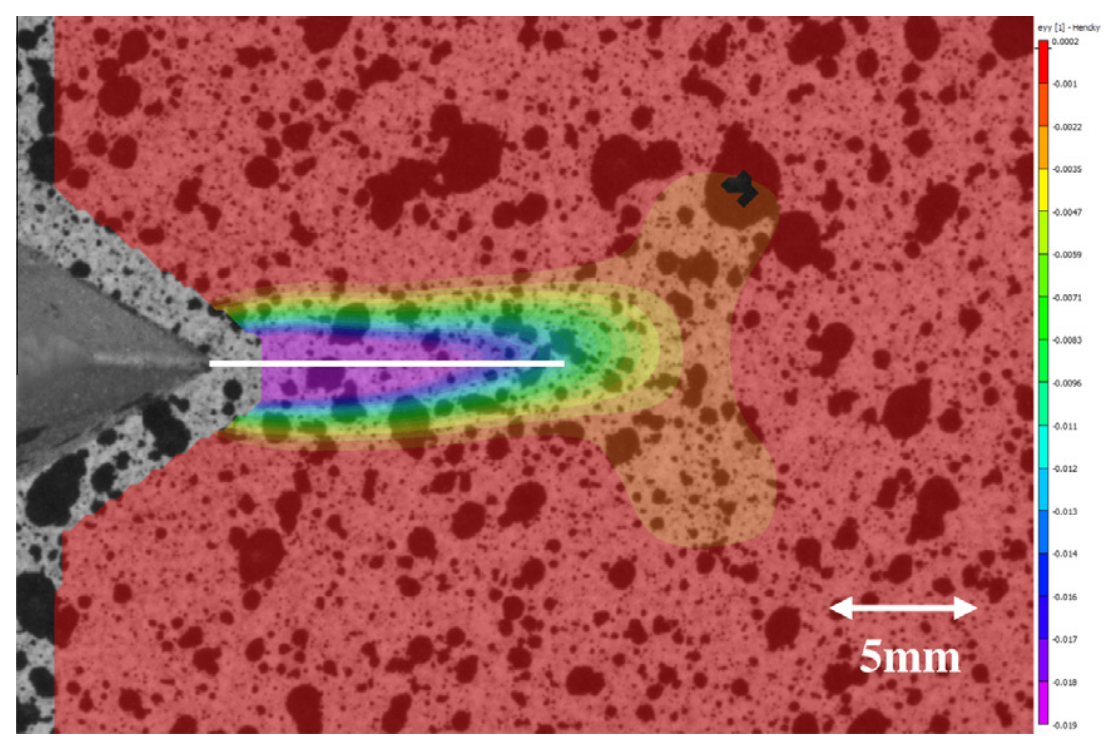

Fig. 11. Contour plot showing compressive strains around crack edges at zero load.

The maximum compressive $e_{y y}$ strain immediately surrounding the crack was 1.9 percent. The compressive strains get progressively smaller as the distance from the crack edges increases. This result compliments very well the results obtained from calculating $K$ from the displacement fields.

As it is observed from the results in Fig. 12, in the early stages of loading, up to almost $4 \mathrm{kN}$ of load, a negative value of mode I stress intensity factor has been extracted from the DIC displacement data. This is simply representative of existing compression stress around the crack edges, as shown in Fig. 11, due to crack closure. Increasing the load separates the crack faces and therefore a positive value is determined as an effective opening mode stress intensity factor.

\subsection{Determining CTOA in the presence of extensive plasticity}

A significant amount of work has also been carried out to characterise displacement fields around a crack during the steady state tearing under monotonic load. The challenge in this situation is that the displacement field is not well characterised by either a conventional linear elastic term, such as $K_{\mathrm{I}}$, or by a non-linear parameter such as $J_{\mathrm{I}}$. Instead, the crack tip opening angle (CTOA) is considered to be a promising approach for characterising ductile fracture where there is significant crack extension [15]. There are various techniques for measuring CTOA [16]. The technique and results presented in this section will be based on displacement field measurements obtained using DIC.

\subsubsection{CTOA fracture criterion}

The CTOA fracture criterion is an evolution of the well established crack tip opening displacement (CTOD) criterion. The basic hypothesis is that for a given alloy with sufficiently large tearing modulus and a degree of hardening, there is a clearly definable crack tip profile and CTOA that is characteristic of the material [17]. The constant CTOA value obtained in the stable phase of crack extension can be used in numerical methods to predict the fracture behaviour of a structure made from the

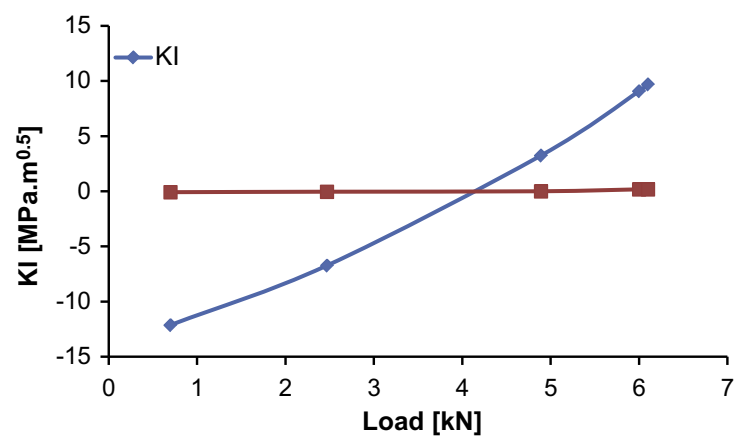

Fig. 12. Measurements of $K$ with increasing load illustrating closure effects. 
same alloy. The constant CTOA value $\left(\mathrm{CTOA}_{\text {material }}\right)$ is considered to be the material fracture resistance and if the crack driving force, measured by the $\mathrm{CTOA}_{\text {applied }}$ of the loaded structure, causes the inequality to be fulfilled, the fracture process will be arrested. However, the fracture process will continue if the inequality is reversed.

\subsubsection{Methodology}

In this work, experiments to determine CTOA from displacement fields was carried out using 2014 aluminium alloy. The geometry of the compact tension (CT) specimen used adhered to the guidelines set in the ASTM E2472 standard [14] which is designed for fracture toughness data under low through thickness constraint conditions. In this case, the low constraint conditions were satisfied by the plate thickness which was $5.0 \mathrm{~mm}$ and the resultant geometry of the specimen is illustrated in Fig. 13. The geometry of the CT specimen gave an initial crack length to width ratio of approximately 0.4 after fatigue precracking which was carried out to obtain a sharp initial crack tip.

To facilitate the use of DIC to obtain displacement field measurements, a random speckle was applied to the surface of the specimen in a $10 \mathrm{~mm}$ thick strip along the entire mid-section of the specimen as shown in Fig. 14 . A grid $(1.0 \times 2.0 \mathrm{~mm})$ was etched onto the surface before the speckle pattern was applied and was used predominantly for crack tracking purposes. The specimens were fractured under mode I conditions in a $100 \mathrm{kN}$ servo hydraulic machine. Loading grips were designed to minimise buckling and twisting of the specimens. The test was conducted under quasi-static conditions in displacement control at a rate of $0.02 \mathrm{~mm} / \mathrm{s}$. Data acquisition was done using the integrated DIC system which allowed all the test parameters to be synchronised.

CTOA was calculated from displacement fields measured using DIC as shown in Fig. 15. Two points, one above the crack tip and one below the crack tip along the same vertical axis, are chosen from which the relative vertical displacement is calculated. The distance to the crack tip is calculated from the coordinates from which CTOA is derived from Eq. (11) below:

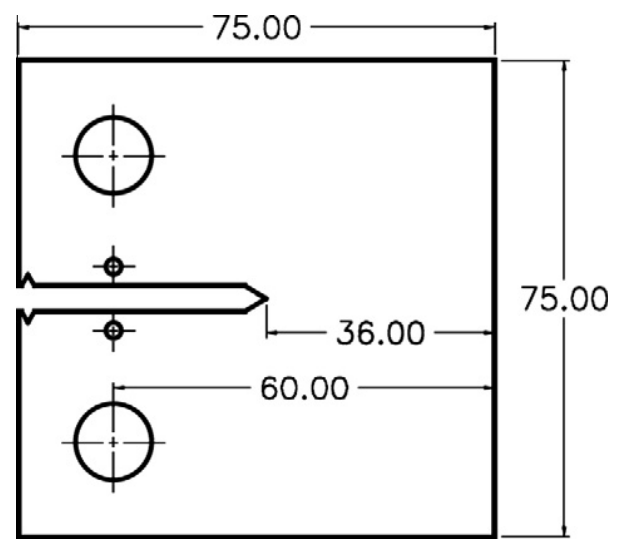

Fig. 13. Schematic of $\mathrm{CT}$ specimen, dimensions in $\mathrm{mm}$.

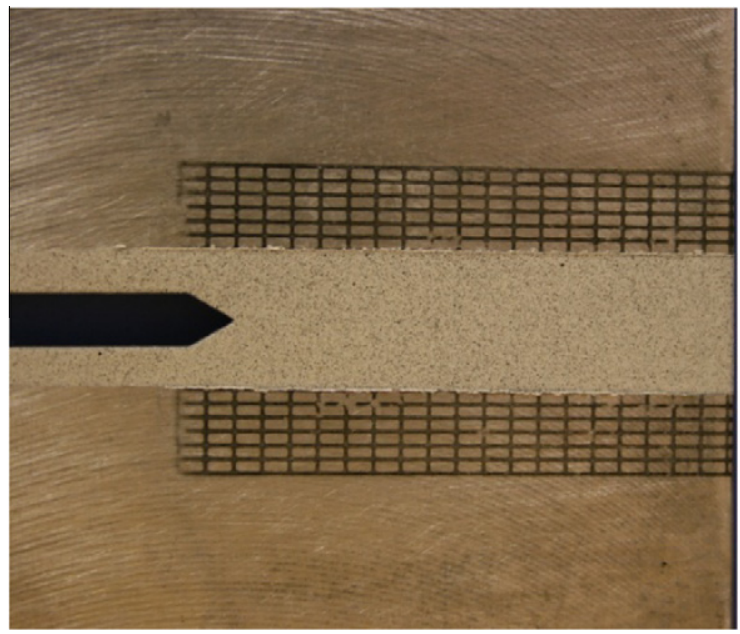

Fig. 14. Prepared specimen with speckle pattern and grid $(1.0 \times 2.0 \mathrm{~mm})$ on the surface. 


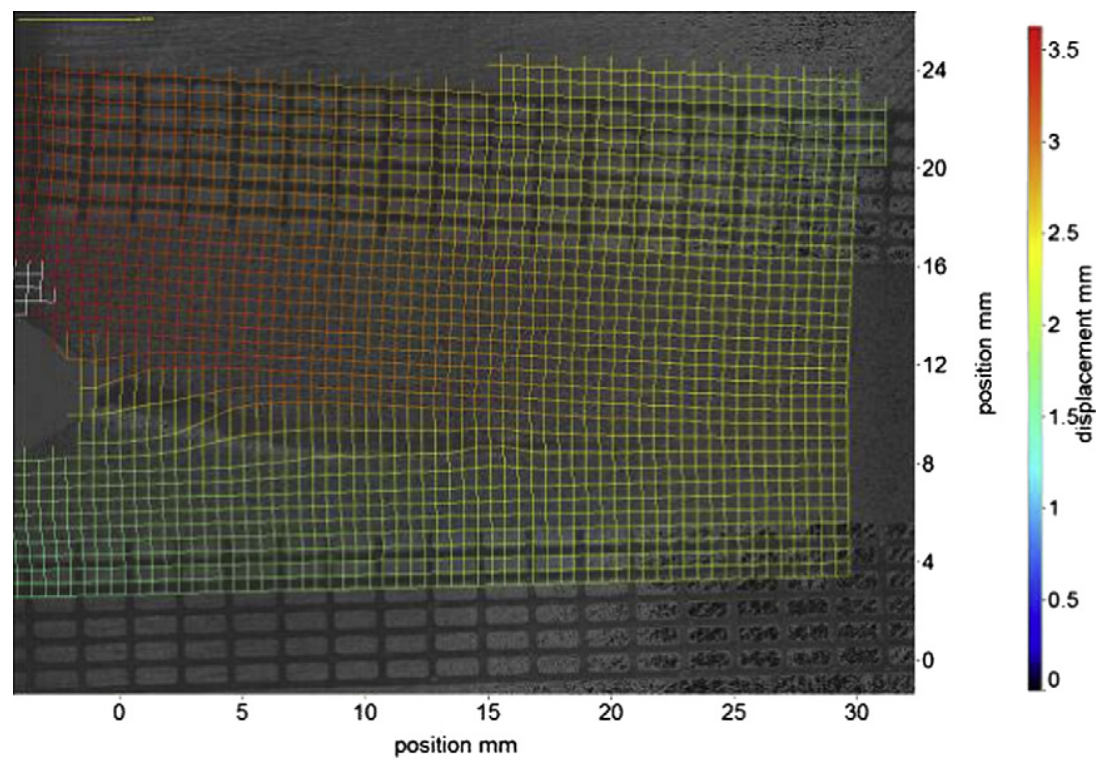

Fig. 15. Displacement fields measured with DIC.

$$
C T O A_{\theta}=2 \tan ^{-1} \frac{\left(V_{b}-V_{a}\right)}{2 A}
$$

where $A=$ distance from crack tip to measurement position, $V_{b}=$ vertical vector displacement below crack tip, $V_{a}=$ vertical vector displacement above crack tip.

\subsubsection{Experimental results}

Displacement fields measured for one particular specimen at different stages of crack extension are shown in Fig. 16. The origin of the $X$ - and $Y$-coordinates correspond to the location of the crack tip and the $X Y$-plane represents the planar surface of the specimen. Values of the vertical displacement are absolute values with rigid body displacements subtracted. There are minimal differences in the displacement field data around the crack tip between the different crack lengths which suggest that there are only small differences in the respective crack geometries. The displacement field data also shows that there is significant plastic deformation around the crack path which is evident from the reduction in the measured displacement values.

From the displacement field data, CTOA values were computed from the corresponding data points using the CTOA calculation methodology explained in the preceding section and the CTOA distribution obtained is shown in Fig. 17 for two different crack lengths, denoted by data labels ' $a$ ' and 'b' corresponding to crack lengths of $7.3 \mathrm{~mm}$ and $12.6 \mathrm{~mm}$ respectively. The numbers in the data labels are the $Y$-coordinates of the vector location on the specimen surface and the crack tip location is at the origin of the $\mathrm{X}$-axis.

Behind the crack tip, the resultant CTOA values clearly indicate that there is a measurable angle which remains relatively unchanged until the corresponding data points approach the crack tip where an increase in CTOA values is observed. This increase is normally observed when the distance between the two corresponding points in the $Y$-direction is bigger than the distance of the crack tip to corresponding measurement points in the $X$-direction. Further details on this can be found in literature [18]. The average CTOA value obtained in the $5-20 \mathrm{~mm}$ region behind the crack tip was $6.23^{\circ}$ which corresponded very well to the values obtained using other more conventional techniques ( $\delta_{5}$ and ASTM DIC method [14]) used to measure CTOA for this particular specimen.

Results from the calculation of CTOA from displacement field measurements have shown that it is possible to obtain consistent CTOA data from full field displacement data measured using DIC. However, adequate measures need to be taken to discriminate data affected by extensive plasticity close to the crack path. The results obtained from both displacement fields and subsequent CTOA values show that for a given material and geometry, there is a measurable profile which is characteristic of the crack geometry. This hypothesis is similar to the basis of the CTOA criterion but characterising this profile using full field measurement techniques such as DIC is less tedious and the level of uncertainty is lower.

\subsection{Preliminary investigation of anisotropic fracture behaviour using 3D DIC}

Anisotropy in the fatigue and fracture behaviour of aluminium lithium alloys remains a concern which has limited its widespread use especially in primary structural components. This section reports on a preliminary investigation into the 


\section{Displacement fields around crack tip for image 215 - crack length $7.3 \mathrm{~mm}$}

(a)

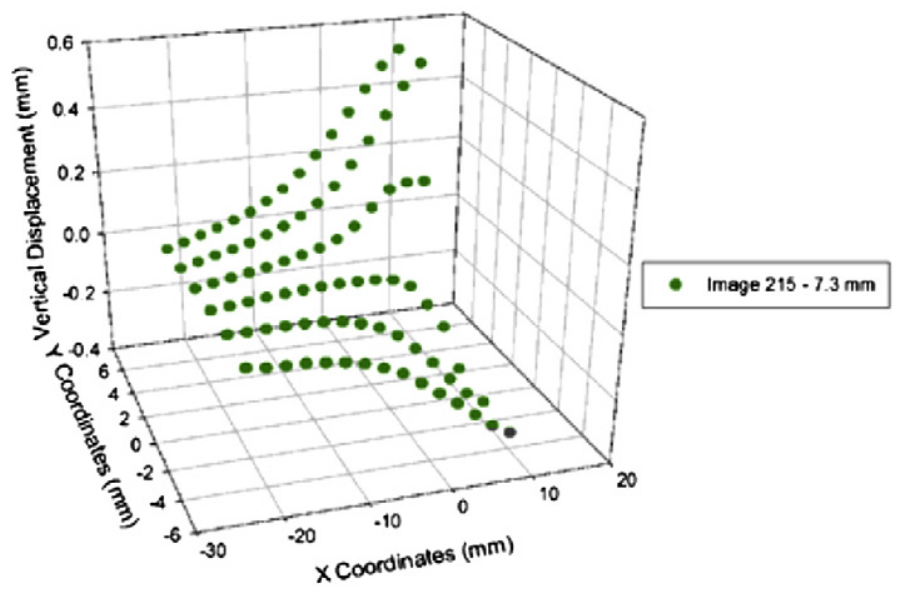

Displacement fields around crack tip for image 252 - crack length $12.6 \mathrm{~mm}$

(b)

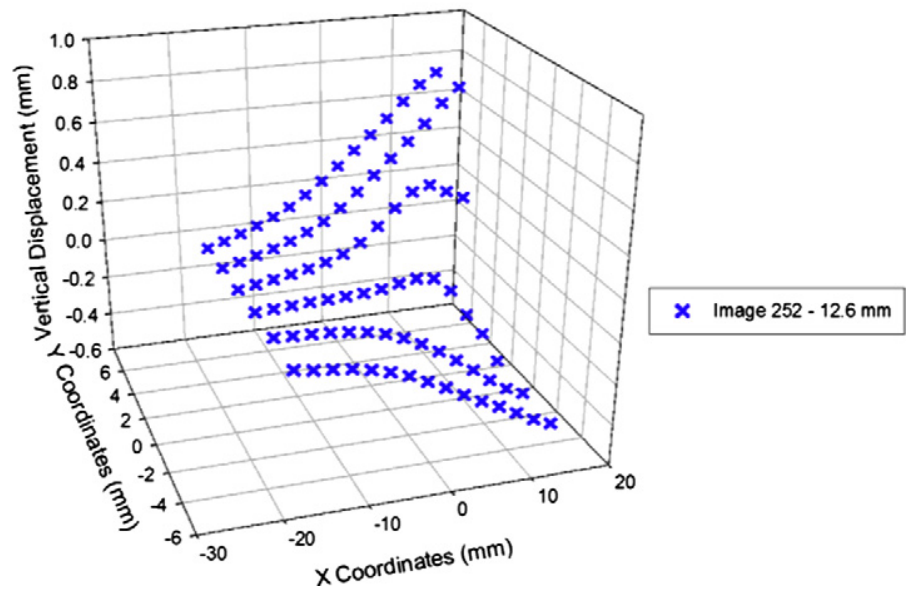

Fig. 16. Displacement fields for different crack lengths.

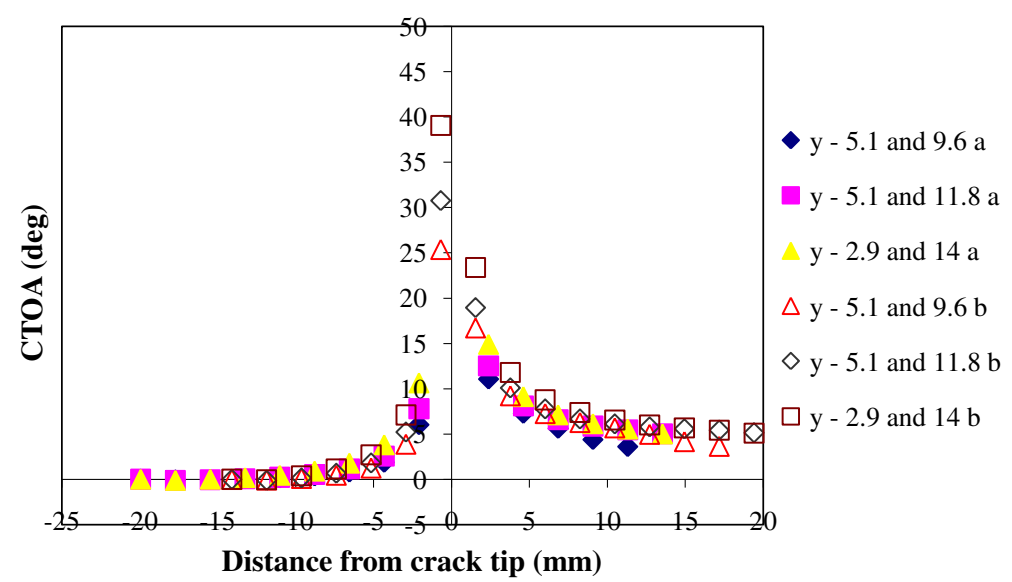

Fig. 17. СTOA values derived from displacement fields for different crack lengths.

fracture behaviour of a fourth generation 2050-T 851 aluminium alloy by using 3D DIC to characterise the deformation of the specimen surface during ductile tearing. It is anticipated that useful parameters can be extracted from the deformation data in the future which could provide better characterisation of fracture parameters. 


\subsubsection{Methodology}

CT specimens were machined from the plate into $10 \mathrm{~mm}$ thick specimens. The design of the specimen was based on the guidelines in the ASTM E2472 standard [14] and had in-plane dimensions of $100 \times 96 \mathrm{~mm}$ which is illustrated in Fig. 10. The specimens were machined from the plate in both TL (crack propagating along rolling direction) and LT (crack propagating across to rolling direction) configuration. The notch was machined by electrode discharge machining (EDM). The specimens were fatigue pre-cracked prior to testing to generate a sharp crack in a bid to minimise any instability at the beginning of the fracture process.

The tests were carried in a servo hydraulic Mayes test frame with hydraulic grips. Bespoke grips were made to hold the specimen and minimise buckling during the quasi-static tearing process. Displacement rate of the actuator was set at $0.05 \mathrm{~mm} / \mathrm{s}$. 3D DIC was used for displacement measurements which required a random speckle pattern to be applied on the surface of the specimens. This was done by spraying the area of interest with white paint which was then speckled using black paint. Image acquisition and data acquisition was done with the Vic3D DIC system.

\subsubsection{Experimental results}

Fig. 18 shows the torn specimens with out of plane deformation maps superimposed on the surface. The crack path of the specimen in the LT direction is highly unstable. There was a significant amount of crack tunnelling and the fracture mode comprised of a combination of slant and shear fracture.

Further evidence of the fracture instability is encountered in the load CMOD plot for the LT specimen shown in Fig. 19. There is a sudden drop in load $(21-7.2 \mathrm{kN})$ which in turn was followed by the crack beginning to turn approximate $45^{\circ}$ as shown in Fig. 18. The corresponding CMOD increases by $0.37 \mathrm{~mm}(1.16-1.53 \mathrm{~mm})$. Preliminary investigation of the fracture surface suggests sudden fracture between grain boundaries to be the most likely cause. The cause of the crack turning has not been established but based on literature data it would most likely be microstructure related [19]. Further analysis of the fracture surfaces and data is needed to determine the cause of this phenomenon.

For the specimen in the TL direction, the crack path was stable. There was minimal amount of crack tunnelling and it was predominantly flat fracture throughout the tearing process with only small shear lips forming at the edges. The tearing resistance was significantly less when compared to the LT direction. This anisotropic fracture behaviour however did not translate into significant differences in the out of plane deformation as shown in Fig. 18. However, being able to measure the size of the plastic zone will lead to more accurate assessment of fracture parameters from the measured displacement field such as the $J$-integral which is currently underway.
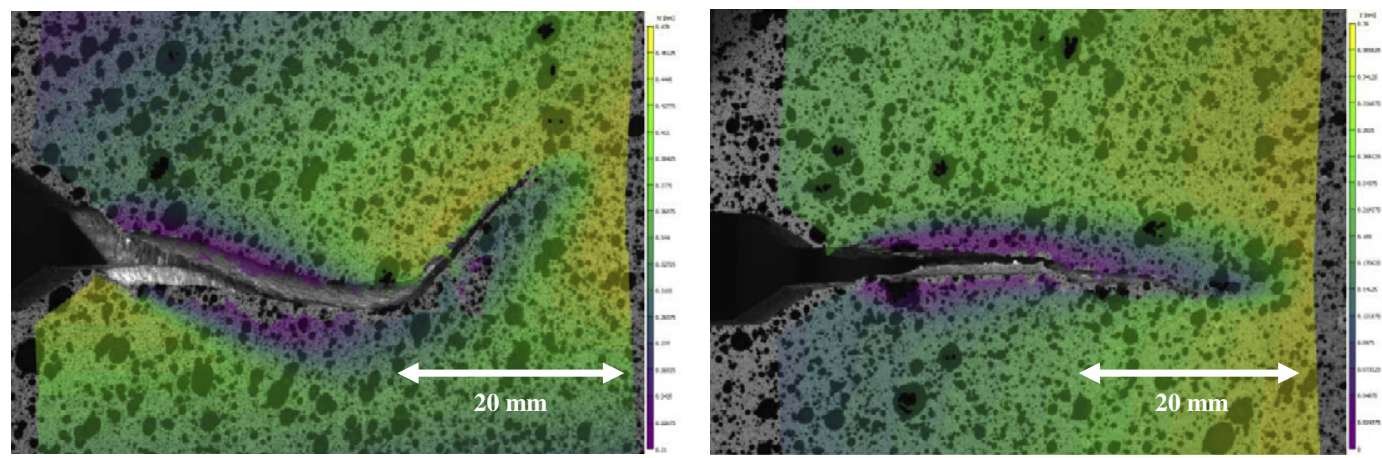

Fig. 18. Out of plane deformation maps of tear specimens, LT (left) and TL (right).

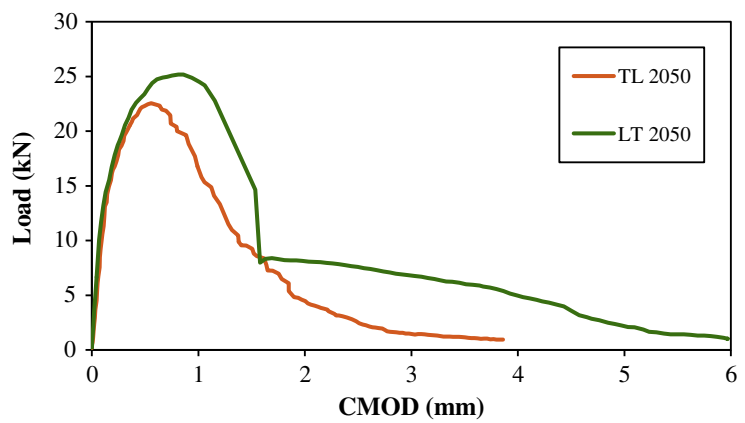

Fig. 19. Load CMOD for 2050 CT tear specimens. 


\section{Concluding discussion}

Direct measurement of the crack tip displacement field under both cyclic and monotonic loading is now readily performed using digital image correlation of pairs of images taken of a cracked structure. Most usefully, the displacement data contains both elastic and plastic contributions.

Analysis of the full field data for a cyclically loaded crack can provide the $K$ and $T$-stress term of the Williams' stress field expansion. This provides valuable experimental information on the effect of geometry on the crack tip constraint and hence the path stability of growing cracks. The technique was also be used to investigate the effects of crack closure.

The ability to capture extensive non-linear crack tip displacements has opened up the possibility of using the crack tip opening angle as a realistic design and assessment parameter for stable ductile tearing. The accurate and extensive data which DIC can produce now enables subtle differences in tearing behaviour to be determined. DIC has proved to be a very useful technique for helping to characterise properties which influence crack paths.

\section{References}

[1] Yates JR, Zanganeh M, Asquith DT, Tai YH. Quantifying crack tip displacement fields: T-stress and CTOA. In: Crack paths. Vicenza, Italy; 2009.

[2] Patterson EA, Olden EJ. Optical analysis of crack tip stress fields: a comparative study. Fatigue Fract Engng Mater Struct 2004;27(7):623-36.

[3] Larsson SG, Carlsson AJ. Influence of non-singular stress terms and specimen geometry on small-scale yielding at crack tips in elastic-plastic materials. J Mech Phys Solids 1973;21:263-77.

[4] Rice JR. Limitations of small scale yielding approximation for crack tip plasticity. Mech Phys Solids 1974;22:17-26.

[5] López-Crespo P, Shterenlikht A, Patterson EA, Yates JR, Withers PJ. Towards real time crack monitoring using image correlation. In: Proceedings of the society for experimental mechanics annual conference and exposition. St. Louis, MO, USA; 2006.

[6] Muskhelishvili NI. Some basic problems of the mathematical theory of elasticity. 4th ed. Leyden, The Netherlands: Noordhoff International; 1977.

[7] Williams ML. On the stress distribution at the base of a stationary crack. J Appl Mech 1957(27):109-14.

[8] Atluri SN, Kobayashi AS. Mechanical responses of materials. In: Kobayashi AS, editor. Handbook on experimental mechanics; 1987. p. 1-40.

[9] Yoneyama S, Morimoto Y, Takashi M. Automatic evaluation of mixed-mode stress intensity factors utilizing digital image correlation. Strain 2006;42(1):21-9.

[10] Vision LA. Davis StrainMaster Software for Davis 7.0; 2005, p. 386.

[11] Zanganeh Gheshlaghi M. Experimental investigation of crack paths. PhD Dept. of Mechanical Engineering, The University of Sheffield; 2008.

[12] ABAQUS Manual, Version 6.6, H. Karlsson and Sorensen Inc.; 2006.

[13] Christopher CJ, James MN, Patterson EA, Tee KF. Towards a new model of crack tip stress fields. Int J Fract 2007;148(4):361-71.

[14] ASTM-E2472. Standard test method for determination of resistance to stable crack extension under low-constraint conditions; 2006.

[15] Newman Jr JC, James MA, Zerbst U. A review of the CTOA/CTOD fracture criterion. Engng Fract Mech 2003;70(3-4):371-85.

[16] Newman Jr JC, Schwalbe K-H. The CTOA and d5 standards for low-constraint fracture specimens. In: 11 th international conference on fracture. Turin, Italy; 2005. p. 6.

[17] Barnby JT, Nadkarni AS, Cresswell SL. Experimental determination of crack tip angles (CTOA) and their applicability to instability prediction in structures. Int J Mater Prod Technol 1995;10(1):161-70.

[18] Tai YH, CTOA measurements in aluminium alloys using direct techniques. PhD Dept. of Mechanical Engineering, University of Sheffield; 2008.

[19] Martins JW. Aluminum-lithium alloys. Annu Rev Mater Sci 1988;18:101-19. 